Nota / Note

\title{
CONTRASTES ORTOGONAIS NA ANÁLISE DO CONTROLE DE VOLATILIZAÇÃO DE AMÔNIA EM COMPOSTAGEM
}

\author{
José Eduardo Corrente*; Maria Cristina Stolf Nogueira; Beatriz Montrágio Costa \\ Depto. de Ciências Exatas - USP/ESALQ, C.P. 9 - CEP: 13418-900 - Piracicaba, SP. \\ ${ }^{*}$ Autor correspondente <jecorren@carpa.ciagri.usp.br>
}

\begin{abstract}
RESUMO: O uso de contrastes ortogonais na análise de experimentos é uma técnica que tem se revelado bastante eficiente na obtenção de efeitos principais, de interação e efeitos aninhados. Em geral, essa técnica é mais útil para a análise de dados de um experimento não convencional, que é aquele que não segue uma estrutura definida. Com esse objetivo, aplicou-se esta técnica aos dados obtidos em um experimento para controlar as perdas de amônia por volatilização com diferentes níveis de acidez residual associados a três aditivos e quatro doses, adicionando uma testemunha. Desse modo, a análise estatística foi conduzida segundo um delineamento inteiramente casualizado numa classificação mista com um tratamento adicional. Os efeitos principais de aditivos e doses, bem como os efeitos aninhados e de interação, foram obtidos através do uso dos contrastes ortogonais. Assim, o uso desta técnica revelou uma forma alternativa para análise de experimento não convencional na obtenção dos efeitos principais, de interação e aninhados.

Palavras-chave: compostagem de esterco, volatilização, tratamento adicional, contraste ortogonal
\end{abstract}

\section{ORTHOGONAL CONTRASTS IN THE ANALYSES OF AMMONIA VOLATILIZATION CONTROL IN COMPOSTING}

\begin{abstract}
Orthogonal contrasts have been very useful in the analysis of experiments and this technique has been efficient in obtaining estimates of main, interaction and nested effects. Generally this technique has been useful for the analysis of data from non-conventional experiments, with no defined structure. The aim of this work was to apply this technique to the data obtained by an experiment to control losses of ammonia by volatilization with different levels of residual acidity associated with three levels of additives, and four rate levels. In this way the statistical analysis was carried out according to a one-way layout in a mixture with an additional treatment. The main effects of additives and rate as well as the nested and interaction effects were estimated using orthogonal contrasts. This technique is an alternative form to analyze non-conventional experiments in order to extract all the effects involved in the analysis.
\end{abstract}

Key words: composting, volatilization, additional treatment, orthogonal contrast

\section{INTRODUÇÃO}

A compostagem é uma importante técnica para o aproveitamento de resíduos orgânicos, muitas vezes obtidos como subproduto da atividade agrícola. Quando adequadamente produzido e aplicado o composto orgânico pode se constituir em material de boa qualidade para melhorar as características físicas e fisicoquímicas do solo.

O processo de compostagem é resultado da atividade de microorganismos que convertem o nitrogênio em $\mathrm{NH}_{3}$ durante a decomposição do material orgânico. Essa liberação, além de diminuir o teor de nitrogênio do composto, pode contribuir para a poluição do ar (Galbally \& Roy, 1983; Moller \& Schieferdecker, 1985).

Para Freney et al. (1988), o processo de perda de nitrogênio é de extrema importância, seja para compostos orgânicos ou para fertilizantes nitrogenados, pois pode variar de 9 a $86 \%$ do $\mathrm{N}$-fertilizante aplicado. Alexander (1977) afirma que a liberação de amônia torna-se mais provável quando o teor do resíduo é maior que $2,4 \%$, pois qualquer quantidade que excede 0 necessário para os microorganismos decomporem o material orgânico será descartada na forma de $\mathrm{NH}_{3}$.

Alcarde \& Rodela (1996) sugerem a hipótese de haver relação entre a eficiência do superfosfato simples e do gesso agrícola, com a acidez residual presente nestes materiais. $O$ controle seria devido à reação do $\mathrm{NH}_{3}$ formado com $\circ \mathrm{H}^{+}$livre proveniente de ácidos remanescentes do ataque da rocha fosfática, sendo que quanto maior a acidez residual, maior o controle das perdas de amônia por volatilização. Isso explicaria os resultados aparentemente contraditórios de vários trabalhos relacionados ao estudo de perdas de nitrogênio nos processos de volatilização.

Existem vários estudos na tentativa de minimizar a perda de amônia no processo de volatilização através da adição de superfosfato simples e/ou gesso agrícola ao composto. Porém, os estudos são contraditórios com relação à eficiência e quantidades a serem utilizadas desses materiais (Prochnow et al., 1998).

Com o objetivo de estudar as perdas de amônia por volatilização com diferentes valores de acidez residual, Prochnow et al. (1998) conduziram um 
experimento em frascos fechados de vidro de 1,6 litro de capacidade, utilizando como material orgânico uma mistura de quantidades iguais de esterco fresco e seco de galinha e de gado, através da adição de superfosfato simples, superfosfato triplo e gesso agrícola. Uma testemunha também foi considerada como sendo a mistura de esterco sem a adição de qualquer aditivo. A quantidade de amônia perdida do esterco por volatilização foi determinada aos $7,14,21,28,35$ e 42 dias e as quantidades totais de $\mathrm{NH}_{3}$ foram calculadas pela soma das quantidades obtidas de cada semana.

Analisando a maneira como os dados foram obtidos do experimento realizado por Prochnow et al. (1998), observam-se níveis diferentes de acidez residual, impossibilitando a aplicação de uma estrutura fatorial completa. Além, disso existe uma testemunha única para todo experimento.

Sendo assim, o presente trabalho tem por objetivo analisar os dados obtidos desse experimento considerando a estrutura de fatorial $3 \times 4$ ( 3 aditivos e 4 doses) e diferentes níveis de acidez residual aninhada aos aditivos e respectivas doses, incluindo a testemunha como um tratamento adicional. Para testar as hipóteses dos efeitos envolvidos no modelo propõem-se:

(1) analisar os dados considerando cada combinação de aditivos, doses e nível de acidez residual como sendo um tratamento, numa estrutura inteiramente ao acaso.

(2) nos tratamentos aplicar a técnica dos contrastes ortogonais, estimando-se assim as somas de quadrados para todos ao efeitos envolvidos.

Tal proposta se justifica pelo fato de que experimentos instalados considerando a testemunha como um tratamento adicional é usual na experimentação agronômica, devido a economia de material e de área experimental. A desvantagem está na dificuldade da análise dos dados, pois a estrutura passa a ser de dados incompletos. Healy (1956) e Gomes (1987) já citam esse tipo de experimento, mas apresentam apenas um esquema de análise sem muitas complicações.

\section{METODOLOGIA}

Os dados obtidos por Prochnow et al. (1998) constaram de 3 aditivos (gesso agrícola $(\mathrm{G})$; superfosfato simples (SS); superfosfato triplo (ST)) em 4 doses $(50,100$, $\left.150,200 \mathrm{~kg} \mathrm{ton}^{-1}\right)$, 2 níveis de acidez residual para cada aditivo e uma testemunha para uma mistura de esterco de galinha e de gado.

Os níveis de acidez residual para o gesso agrícola foram de $0,13 \%$ e $0,20 \%$; para o superfosfato simples foram de $7,02 \%$ e $2,36 \%$; para o superfosfato triplo foram de $2,38 \%$ e $1,86 \%$.

Os dados observados da quantidade de amônia perdida do esterco por volatilização correspondem ao total das quantidades obtidas no período de 42 dias, com 4 repetições.
Devido a presença da testemunha considerada como um tratamento adicional, e os dados não se encaixarem em uma estrutura de análise convencional, adotou-se cada combinação de aditivos, doses e níveis de acidez residual como um tratamento. Desse modo, pôde-se considerar um modelo matemático de classificação simples inteiramente casualizado com 25 tratamentos (24 combinações e a testemunha) cuja representação é dada por

$$
y_{i j}=\mu+t_{i}+e_{i j}
$$

com $\mathrm{i}=1, \ldots, 25 \mathrm{e} \mathrm{j}=1, \ldots, 4$, sendo $\mathrm{y}_{\mathrm{ij}}$ o valor observado referente ao tratamento i na repetição j; $\mu$ uma constante; $t_{i} 0$ efeito do tratamento $i$ e $e_{i j} o$ erro experimental. $O$ esquema da análise da variância é dado na TABELA 1.

Neste caso, os graus de liberdade de tratamentos podem ser decompostos através de comparações planejadas de acordo com o interesse do pesquisador em testar efeitos específicos do experimento. Desse modo, para os dados considerados as comparações podem ser construídas com o objetivo de testar os efeitos principais (aditivos e doses), o efeito da interação (aditivos com doses) e os efeitos aninhados de acidez com cada aditivo e dose. Tais comparações podem ser feitas através da técnica de contrastes ortogonais.

Define-se um contrastes como uma função linear estimável da forma

$$
\mathrm{Y}=\sum_{\mathrm{i}=1}^{\mathrm{l}} \mathrm{c}_{\mathrm{i}} \mu_{\mathrm{i}}
$$

sendo $c_{i}$ o coeficiente a ser atribuído a $\mu_{\mathrm{i}} ; \mu_{\mathrm{i}}$ a média do tratamento i, tal que

$$
\sum_{i=1}^{1} c_{i}=0
$$

Dois contrates $Y_{h}=\sum c_{h i} \mu_{i}$ e $y_{h}=\sum c_{h^{\prime} j} \mu_{i}$ com $h \neq h^{\prime}$ para $h=1, \ldots, l-1$, são ortogonais quando $\left\langle\mathrm{c}_{\mathrm{hi}}, \mathrm{c}_{\mathrm{h}^{\prime} \mathrm{i}}\right\rangle=0$

Cada contraste gera uma hipótese a ser testada e está associado a uma soma de quadrados com 1 grau de liberdade. A soma de quadrados para um contrate $Y_{h}$ é dada por

$$
S Q Y_{h}=\frac{r Y_{h}^{2}}{\sum_{i=1}^{1} c_{h i}^{2}}
$$

para $h=1, \ldots, \mathrm{l}-1$, sendo que $\mathrm{Y}_{\mathrm{h}}=\sum \mathrm{c}_{\mathrm{hi}} \overline{\mathrm{y}}_{\mathrm{i}}$. é um estimador não tendencioso de $Y_{h}$. Devido a ortogonalidade dos contrastes ocorre que o: $\sum S Q Y_{h}$ é a soma de quadrados de tratamentos com (I-1) graus de liberdade.

Inicialmente, com a aplicação dessa técnica, para os dados em questão, construiu-se contrastes ortogonais de tal maneira que foi possível testar os efeitos da testemunha vs. os demais tratamentos, de 
aditivos, de doses, da interação de aditivos com doses, e da acidez residual aninhada a cada aditivo e doses. Na TABELA 2 é mostrada a composição dos contrastes.

Como os níveis do fator doses são quantitativos, os coeficientes dos contrastes referentes às doses foram atribuídos de acordo com os coeficientes para interpolação de polinômios ortogonais, segundo Gomes (1987).

Se o efeito da interação de aditivos com doses for significativa, e o efeito da acidez residual aninhada a cada aditivo e doses for não significativo, o passo seguinte é a decomposição da soma dos graus de

TABELA 1 - Esquema da análise da variância, considerando uma classificação simples, para os dados de volatilização.

\begin{tabular}{ll}
\hline \multicolumn{1}{c}{ Causa de Variação } & GL \\
\hline Tratamentos & 24 \\
Resíduo & 75 \\
\hline Total & 99
\end{tabular}

liberdade da interação aditivos com doses com os graus de liberdade de doses, gerando os efeitos referentes as doses aninhada a cada aditivo. Para a estimação desses efeitos, foram construídos os contrastes que são apresentados na TABELA 3.

Os resultados das análises foram obtidos através da aplicação do PROC GLM/SAS (1990).

\section{RESULTADOS E DISCUSSÃO}

Segundo o modelo inicialmente adotado como uma classificação simples inteiramente casualizado com 25 tratamentos e 4 repetições para os dados em questão, a TABELA 4 mostra os resultados da análise da variância inicial.

Houve efeito significativo para os tratamentos na forma como foram considerados. Devido a esse fato, foi feita a decomposição dos 24 graus de liberdade de tratamentos através dos contrastes ortogonais, sugeridos na TABELA 2, cujos resultados obtidos constam na TABELA 5.

TABELA 2 - Tabela dos contrastes ortogonais para efeitos de doses, interação e aninhados.

\begin{tabular}{|c|c|c|c|c|c|c|c|c|c|c|c|c|c|c|c|c|c|c|c|c|c|c|c|c|c|c|}
\hline \multicolumn{3}{|c|}{ Tratamentos } & \multicolumn{24}{|c|}{ Contrastes } \\
\hline Aditivos & Doses & Ac. Res. (\%) & 1 & 2 & 3 & 4 & 5 & 6 & 7 & 8 & 9 & 10 & 11 & 12 & 13 & 14 & 15 & 16 & 17 & 18 & 19 & 20 & 21 & 22 & 23 & 24 \\
\hline Sem & 0 & 0,00 & 24 & 0 & 0 & 0 & 0 & 0 & 0 & 0 & 0 & 0 & 0 & 0 & 0 & 0 & 0 & 0 & 0 & 0 & 0 & 0 & 0 & 0 & 0 & 0 \\
\hline $\mathrm{G}$ & 50 & 0,13 & -1 & 2 & 0 & -3 & 1 & -1 & -6 & 2 & -2 & 0 & 0 & 0 & 1 & 0 & 0 & 0 & 0 & 0 & 0 & 0 & 0 & 0 & 0 & 0 \\
\hline$G$ & 100 & 0,13 & -1 & 2 & 0 & -1 & -1 & 3 & -2 & -2 & 6 & 0 & 0 & 0 & 0 & 1 & 0 & 0 & 0 & 0 & 0 & 0 & 0 & 0 & 0 & 0 \\
\hline $\mathrm{G}$ & 150 & 0,13 & -1 & 2 & 0 & 1 & -1 & -3 & 2 & -2 & -6 & 0 & 0 & 0 & 0 & 0 & 1 & 0 & 0 & 0 & 0 & 0 & 0 & 0 & 0 & 0 \\
\hline$G$ & 200 & 0,13 & -1 & 2 & 0 & 3 & 1 & 1 & 6 & 2 & 2 & 0 & 0 & 0 & 0 & 0 & 0 & 1 & 0 & 0 & 0 & 0 & 0 & 0 & 0 & 0 \\
\hline$G$ & 50 & 0,20 & -1 & 2 & 0 & -3 & 1 & -1 & -6 & 2 & -2 & 0 & 0 & 0 & -1 & 0 & 0 & 0 & 0 & 0 & 0 & 0 & 0 & 0 & 0 & 0 \\
\hline$G$ & 100 & 0,20 & -1 & 2 & 0 & -1 & -1 & 3 & -2 & -2 & 6 & 0 & 0 & 0 & 0 & -1 & 0 & 0 & 0 & 0 & 0 & 0 & 0 & 0 & 0 & 0 \\
\hline$G$ & 150 & 0,20 & -1 & 2 & 0 & 1 & -1 & -3 & 2 & -2 & -6 & 0 & 0 & 0 & 0 & 0 & -1 & 0 & 0 & 0 & 0 & 0 & 0 & 0 & 0 & 0 \\
\hline $\mathrm{G}$ & 200 & 0,20 & -1 & 2 & 0 & 3 & 1 & 1 & 6 & 2 & 2 & 0 & 0 & 0 & 0 & 0 & 0 & -1 & 0 & 0 & 0 & 0 & 0 & 0 & 0 & 0 \\
\hline SS & 50 & 7,02 & -1 & -1 & 1 & -3 & 1 & -1 & 3 & -1 & 1 & -3 & 1 & -1 & 0 & 0 & 0 & 0 & 1 & 0 & 0 & 0 & 0 & 0 & 0 & 0 \\
\hline SS & 100 & 7,02 & -1 & -1 & 1 & -1 & -1 & 3 & 1 & 1 & -3 & -1 & -1 & 3 & 0 & 0 & 0 & 0 & 0 & 1 & 0 & 0 & 0 & 0 & 0 & 0 \\
\hline SS & 150 & 7,02 & -1 & -1 & 1 & 1 & -1 & -3 & -1 & 1 & 3 & 1 & -1 & -3 & 0 & 0 & 0 & 0 & 0 & 0 & 1 & 0 & 0 & 0 & 0 & 0 \\
\hline SS & 200 & 02 & -1 & -1 & 1 & 3 & 1 & 1 & -3 & -1 & -1 & 3 & 1 & 1 & 0 & 0 & 0 & 0 & 0 & 0 & 0 & 1 & 0 & 0 & 0 & 0 \\
\hline SS & 50 & 2,36 & -1 & -1 & 1 & -3 & 1 & -1 & 3 & -1 & 1 & -3 & 1 & -1 & 0 & 0 & 0 & 0 & -1 & 0 & 0 & 0 & 0 & 0 & 0 & 0 \\
\hline SS & 100 & 2,36 & -1 & -1 & 1 & -1 & -1 & 3 & 1 & 1 & -3 & -1 & -1 & 3 & 0 & 0 & 0 & 0 & 0 & -1 & 0 & 0 & 0 & 0 & 0 & 0 \\
\hline SS & 150 & 2,36 & -1 & -1 & 1 & 1 & -1 & -3 & -1 & 1 & 3 & 1 & -1 & -3 & 0 & 0 & 0 & 0 & 0 & 0 & -1 & 0 & 0 & 0 & 0 & 0 \\
\hline SS & 200 & 2,36 & -1 & -1 & 1 & 3 & 1 & 1 & -3 & -1 & -1 & 3 & 1 & 1 & 0 & 0 & 0 & 0 & 0 & 0 & 0 & -1 & 0 & 0 & 0 & 0 \\
\hline ST & 50 & 2,38 & -1 & -1 & -1 & -3 & 1 & -1 & 3 & -1 & 1 & 3 & -1 & 1 & 0 & 0 & 0 & 0 & 0 & 0 & 0 & 0 & 1 & 0 & 0 & 0 \\
\hline ST & 100 & 2,38 & -1 & -1 & -1 & -1 & -1 & 3 & 1 & 1 & -3 & 1 & 1 & -3 & 0 & 0 & 0 & 0 & 0 & 0 & 0 & 0 & 0 & 1 & 0 & 0 \\
\hline ST & 150 & 2,38 & -1 & -1 & -1 & 1 & -1 & -3 & -1 & 1 & 3 & -1 & 1 & 3 & 0 & 0 & 0 & 0 & 0 & 0 & 0 & 0 & 0 & 0 & 1 & 0 \\
\hline ST & 200 & 2,38 & -1 & -1 & -1 & 3 & 1 & 1 & -3 & -1 & -1 & -3 & -1 & -1 & 0 & 0 & 0 & 0 & 0 & 0 & 0 & 0 & 0 & 0 & 0 & 1 \\
\hline ST & 50 & 1,86 & -1 & -1 & -1 & -3 & 1 & -1 & 3 & -1 & 1 & 3 & -1 & 1 & 0 & 0 & 0 & 0 & 0 & 0 & 0 & 0 & -1 & 0 & 0 & 0 \\
\hline ST & 100 & 1,86 & -1 & -1 & -1 & -1 & -1 & 3 & 1 & 1 & -3 & 1 & 1 & -3 & 0 & 0 & 0 & 0 & 0 & 0 & 0 & 0 & 0 & -1 & 0 & 0 \\
\hline ST & 150 & 1,86 & -1 & -1 & -1 & 1 & -1 & -3 & -1 & 1 & 3 & -1 & 1 & 3 & 0 & 0 & 0 & 0 & 0 & 0 & 0 & 0 & 0 & 0 & -1 & 0 \\
\hline ST & 200 & 1,86 & -1 & -1 & -1 & 3 & 1 & 1 & -3 & -1 & -1 & -3 & -1 & -1 & 0 & 0 & 0 & 0 & 0 & 0 & 0 & 0 & 0 & 0 & 0 & -1 \\
\hline CONT & ZASTES: & & 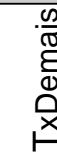 & $\overline{0}$ & $\frac{N}{4}$ & 5 & ธิ & อ & & & & & & & 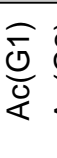 & 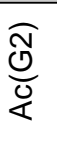 & 感 & 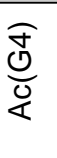 & $\begin{array}{c}\widehat{\bar{c}} \\
\stackrel{\tilde{U}}{\mathcal{U}}\end{array}$ & $\begin{array}{l}\text { तु } \\
\text { ल) } \\
\stackrel{0}{0}\end{array}$ & $\begin{array}{l}\text { लू } \\
\text { ल } \\
\stackrel{0}{0}\end{array}$ & $\begin{array}{l}\overparen{J} \\
\text { D } \\
\stackrel{0}{0}\end{array}$ & 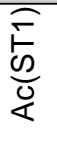 & 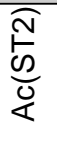 & $\begin{array}{l}\widehat{m} \\
\stackrel{\mathscr{D}}{0} \\
\stackrel{0}{<}\end{array}$ & 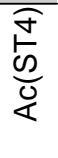 \\
\hline & & & 1 & 2 & 3 & 4 & 5 & 6 & 7 & 8 & 9 & 10 & 11 & 12 & 131 & 14 & & 16 & & & 19 & & & 2 & 23 & 24 \\
\hline EFEIT & & & & Aditi & ivos & & Dose & & & Adit & IIVOS & $5 \mathrm{XUO}$ & 000 & & & Acid & dezu & & & tores & $2(0)$ & & & Fitue & $5<10$ & \\
\hline & & & $\sqrt{2}$ & Z & & & I & & & & 5 & $\square$ & & & & & Л & & & & Z & & & & $\Omega$ & \\
\hline GRAU & DE LIBEF & DADE: & $1 \mathrm{gl}$ & $2 q$ & & & $3 g l$ & & & & 6 & $g \mid$ & & & & & $\mathrm{tgl}$ & & & & tgl & & & & $4 \mathrm{gl}$ & \\
\hline
\end{tabular}


TABELA 3 - Tabela dos contrastes ortogonais para a decomposição da interação.

\begin{tabular}{|c|c|c|c|c|c|c|c|c|c|c|c|c|c|c|c|c|c|c|c|c|c|c|c|c|}
\hline \multicolumn{3}{|c|}{ Tratamentos } & \multicolumn{22}{|c|}{ Contrastes } \\
\hline Aditivos & Doses & Ac. Res. (\%) & 1 & 2 & 3 & 4 & 5 & 6 & 7 & 8 & 9 & 10 & 11 & 12 & 13 & 1415 & 16 & 17 & 181 & 192 & 2021 & 22 & 23 & 24 \\
\hline Sem & 0 & 0,00 & 24 & 0 & 0 & 0 & 0 & 0 & 0 & 0 & 0 & 0 & 0 & 0 & 0 & 0 & 0 & 0 & 0 & 00 & 00 & 0 & 0 & 0 \\
\hline $\mathrm{G}$ & 100 & 0,13 & -1 & 2 & 0 & 3 & -2 & -2 & 0 & 0 & 0 & 0 & 0 & 0 & 0 & 1 & 0 & 0 & 0 & 0 & 0 & 0 & 0 & 0 \\
\hline $\mathrm{G}$ & 150 & 0,13 & -1 & 2 & 0 & -3 & 2 & -2 & 0 & 0 & 0 & 0 & 0 & 0 & 0 & 0 & 0 & 0 & 0 & 0 & 0 & 0 & 0 & 0 \\
\hline $\mathrm{G}$ & 200 & 0,13 & -1 & 2 & 0 & 1 & 6 & 2 & 0 & 0 & 0 & 0 & 0 & 0 & 0 & 0 & 1 & 0 & 0 & 0 & 0 & 0 & 0 & 0 \\
\hline$G$ & 50 & 0,20 & -1 & 2 & 0 & -1 & -6 & 2 & 0 & 0 & 0 & 0 & 0 & 0 & -1 & 0 & 0 & 0 & 0 & 0 & 0 & 0 & 0 & 0 \\
\hline $\mathrm{G}$ & 100 & 0,20 & -1 & 2 & 0 & 3 & -2 & -2 & 0 & 0 & 0 & 0 & 0 & 0 & 0 & -1 & 0 & 0 & 0 & 0 & 0 & 0 & 0 & 0 \\
\hline $\mathrm{G}$ & 150 & 0,20 & -1 & 2 & 0 & -3 & 2 & -2 & 0 & 0 & 0 & 0 & 0 & 0 & 0 & -1 & 0 & 0 & 0 & 0 & 0 & 0 & 0 & 0 \\
\hline $\mathrm{G}$ & 200 & 0,20 & -1 & 2 & 0 & 1 & 6 & 2 & 0 & 0 & 0 & 0 & 0 & 0 & 0 & 0 & -1 & 0 & 0 & 0 & 0 & 0 & 0 & 0 \\
\hline SS & 50 & 7,02 & -1 & -1 & 1 & 0 & 0 & 0 & -1 & -6 & 2 & 0 & 0 & 0 & 0 & 0 & 0 & 1 & 0 & 0 & 0 & 0 & 0 & 0 \\
\hline SS & 100 & 7,02 & -1 & -1 & 1 & 0 & 0 & 0 & 3 & -2 & -2 & 0 & 0 & 0 & 0 & 0 & 0 & 0 & 1 & 0 & 0 & 0 & 0 & 0 \\
\hline SS & 150 & 7,02 & -1 & -1 & 1 & 0 & 0 & 0 & -3 & 2 & -2 & 0 & 0 & 0 & 0 & 0 & 0 & 0 & 0 & 1 & 0 & 0 & 0 & 0 \\
\hline SS & 200 & 7,02 & -1 & -1 & 1 & 0 & 0 & 0 & 1 & 6 & 2 & 0 & 0 & 0 & 0 & 0 & 0 & 0 & 0 & 0 & 0 & 0 & 0 & 0 \\
\hline SS & 50 & 2,36 & -1 & -1 & 1 & 0 & 0 & 0 & -1 & -6 & 2 & 0 & 0 & 0 & 0 & 0 & 0 & -1 & 0 & 0 & 0 & 0 & 0 & 0 \\
\hline SS & 100 & 2,36 & -1 & -1 & 1 & 0 & 0 & 0 & 3 & -2 & -2 & 0 & 0 & 0 & 0 & 0 & 0 & 0 & -1 & 0 & 0 & 0 & 0 & 0 \\
\hline SS & 150 & 2,36 & -1 & -1 & 1 & 0 & 0 & 0 & -3 & 2 & -2 & 0 & 0 & 0 & 0 & 0 & 0 & 0 & 0 & -1 & 0 & 0 & 0 & 0 \\
\hline SS & 200 & 2,36 & -1 & -1 & 1 & 0 & 0 & 0 & 1 & 6 & 2 & 0 & 0 & 0 & 0 & 0 & 0 & 0 & 0 & 0 & 0 & 0 & 0 & 0 \\
\hline ST & 50 & 2,38 & -1 & -1 & -1 & 0 & 0 & 0 & 0 & 0 & 0 & -1 & -6 & 2 & 0 & 0 & 0 & 0 & 0 & 0 & 1 & 0 & 0 & 0 \\
\hline ST & 100 & 2,38 & -1 & -1 & -1 & 0 & 0 & 0 & 0 & 0 & 0 & 3 & -2 & -2 & 0 & 0 & 0 & 0 & 0 & 0 & 0 & 1 & 0 & 0 \\
\hline ST & 150 & 2,38 & -1 & -1 & -1 & 0 & 0 & 0 & 0 & 0 & 0 & -3 & 2 & -2 & 0 & 0 & 0 & 0 & 0 & 0 & 0 & 0 & 1 & 0 \\
\hline ST & 200 & 2,38 & -1 & -1 & -1 & 0 & 0 & 0 & 0 & 0 & 0 & 1 & 6 & 2 & 0 & 0 & 0 & 0 & 0 & 0 & 0 & 0 & 0 & 1 \\
\hline ST & 50 & 1,86 & -1 & -1 & -1 & 0 & 0 & 0 & 0 & 0 & 0 & -1 & -6 & 2 & 0 & 0 & 0 & 0 & 0 & 0 & -1 & 0 & 0 & 0 \\
\hline ST & 100 & 1,86 & -1 & -1 & -1 & 0 & 0 & 0 & 0 & 0 & 0 & 3 & -2 & -2 & 0 & 0 & 0 & 0 & 0 & 0 & 0 & -1 & 0 & 0 \\
\hline ST & 150 & 1,86 & -1 & -1 & -1 & 0 & 0 & 0 & 0 & 0 & 0 & -3 & 2 & -2 & 0 & 0 & 0 & 0 & 0 & 0 & 0 & 0 & -1 & 0 \\
\hline ST & 200 & 1,86 & -1 & -1 & -1 & 0 & 0 & 0 & 0 & 0 & 0 & 1 & 6 & 2 & 0 & 0 & 0 & 0 & 0 & 0 & 0 & 0 & 0 & -1 \\
\hline CONTR & ASTES: & & 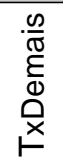 & $\frac{7}{8}$ & $\frac{N}{0}$ & $\frac{\widehat{\sigma}}{\stackrel{0}{\Delta}}$ & $\begin{array}{l}\text { ত্ } \\
\text { ত্ }\end{array}$ & ত্তি & $\frac{\widehat{\bar{c}}}{\stackrel{\oplus}{\square}}$ & $\begin{array}{l}\widehat{\widetilde{N}} \\
\stackrel{\mathscr{N}}{\square}\end{array}$ & $\begin{array}{l}\widehat{\infty} \\
\stackrel{\infty}{\omega}\end{array}$ & $\frac{\widehat{E}}{\mathscr{C}}$ & $\frac{\overparen{N}}{\stackrel{\mathscr{C}}{D}}$ & & 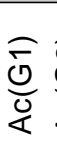 & 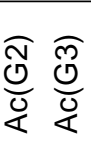 & $\frac{\overparen{J}}{\stackrel{J}{0}}$ & 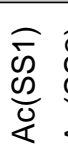 & 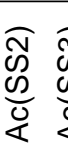 & 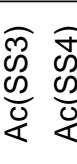 & 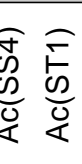 & $\frac{\overparen{N}}{\frac{\omega}{\sigma}}$ & $\frac{\widehat{m}}{\stackrel{\infty}{c}}$ & $\frac{\overparen{J}}{\frac{\mathscr{C}}{0}}$ \\
\hline & & & 1 & & 3 & 4 & 5 & 6 & 7 & 8 & 9 & 10 & & & & 1415 & 16 & 171 & & & 2021 & & 23 & \\
\hline EFEITO & & & & Aditiv & & & $\mathrm{se}(\mathrm{C}$ & & & & & & se(S & & & $\operatorname{cidez}(\mathrm{G}$ & & & idez( & (SS) & & ה & $\mathrm{ez}(\mathrm{S})$ & \\
\hline & & & 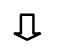 & 16 & & & 17 & & & I & & & $\Omega$ & & & 16 & & & $\sqrt{7}$ & & & & 4 & \\
\hline GRAU & E LIBER & ADE: & $1 \mathrm{gl}$ & $2 \mathrm{gl}$ & & & $3 g l$ & & & $3 \mathrm{gl}$ & & & $3 \mathrm{gl}$ & & & $4 \mathrm{gl}$ & & & $4 \mathrm{gl}$ & & & $4 g$ & & \\
\hline
\end{tabular}

A testemunha difere estatisticamente dos demais tratamentos (TABELA 5). Além disso, verifica-se significância para o efeito da interação de aditivos com doses e para o efeito aninhado de acidez residual com aditivos e doses. Devido a este fato efetuou-se a sua decomposição nos efeitos de acidez residual aninhado para gesso agrícola, superfosfato simples e superfosfato triplo, cujos resultados são apresentados na TABELA 6, observando-se efeito significativo de acidez residual na presença de doses de superfosfato simples.

Como o efeito da interação aditivos com doses foi significativo, procedeu-se à decomposição dos 9 graus de liberdade, referente a adição dos 6 graus de liberdade da interação aditivos com doses com os 3 graus de liberdade de doses, de acordo com a TABELA 3. Os resultados obtidos são apresentados na TABELA 6 .

Constatou-se efeito significativo para os efeitos de doses de superfosfato simples e de superfosfato triplo (TABELA 6). Como foi constatado efeito significativo de acidez residual na presença de doses de superfosfato simples, procurou-se estudar os efeitos linear, quadrático e cúbico para as doses de superfosfato triplo. E também, o efeito da acidez residual aninhada em cada dose de superfosfato simples, cujos resultados encontram-se na TABELA 7.

Constatou-se efeito quadrático significativo para as doses na presença de superfosfato triplo (TABELA 7), revelando assim que a volatilização da amônia em função de doses crescentes de superfosfato triplo pode ser explicado pela equação de regressão apresentada na Figura 1.

Continuando com a análise dos resultados apresentados na TABELA 7, também, constatou-se, através da aplicação do teste $F$ ao nível de $5 \%$ de significância, efeito significativo de acidez residual na presença da dose $100 \mathrm{~kg}_{\text {ton }}{ }^{-1}$ de superfosfato simples, cujos resultados obtidos foram:

\begin{tabular}{ccc}
\hline Acidez Residual & Doses de SS & Média \\
\hline$\%$ & $\mathrm{~kg} \mathrm{t}^{-1}$ & \\
7,02 & 100 & $16,625 \mathrm{a}$ \\
2,36 & 100 & $38,395 \mathrm{~b}$ \\
\hline
\end{tabular}


TABELA 4 - Análise da variância inicial.

\begin{tabular}{lcrrrc}
\hline Fonte de variação & G.L. & S.Q. & Q.M. & F & p-valor \\
\hline Tratamentos & 24 & 18260,3231 & 760,8468 & 14,7400 & 0,0001 \\
Resíduo & 75 & 3870,9259 & 51,6123 & & \\
\hline Total & 99 & 22131,2491 & & & \\
\hline
\end{tabular}

TABELA 5 - Análise da variância obtida através da decomposição dos 24 graus de liberdade de tratamentos através de contrastes ortogonais.

\begin{tabular}{lcrrrr}
\hline Fonte de Variação & G.L. & \multicolumn{1}{c}{ S.Q. } & Q.M. & \multicolumn{1}{c}{ F } & p-valor \\
\hline Test. vs. demais & 1 & 705,4239 & 705,4239 & 13,6700 & 0,0004 \\
Aditivos & 2 & 10944,8960 & 5472,4480 & 106,0300 & 0,0001 \\
Doses & 3 & 3434,5840 & 1144,8613 & 22,1800 & 0,0001 \\
Aditivos x Doses & 6 & 4701,7439 & 783,6240 & 15,1800 & 0,0001 \\
Acidez (Aditivos x Doses ) & 12 & 1646,5568 & 137,2131 & 2,6585 & 0,0050 \\
Resíduo & 75 & 3870,9259 & 51,6123 & & \\
\hline Total & 99 & 22131,2491 & & & \\
\hline
\end{tabular}

TABELA 6 - Análise da variância apresentando os efeitos de doses para cada aditivo e os efeitos de acidez residual aninhado para cada aditivo.

\begin{tabular}{lcrrrr}
\hline Fonte de Variação & G.L. & \multicolumn{1}{c}{ S.Q. } & \multicolumn{1}{c}{ Q.M. } & \multicolumn{1}{c}{ F } & p-valor \\
\hline Test. vs. demais & 1 & 705,4239 & 705,4239 & 13,6700 & 0,0004 \\
Aditivos & 2 & 10944,8960 & 5472,4480 & 106,0300 & 0,0001 \\
\hline Doses (G) & 3 & 110,7075 & 36,9025 & 0,7100 & 0,5461 \\
\hline Doses (SS) & 3 & 3516,8991 & 1172,2997 & 22,7100 & 0,0001 \\
Doses (ST) & 3 & 1335,8398 & 445,2799 & 8,6300 & 0,0001 \\
Doses (Aditivos) & 9 & 4963,4464 & 551,4940 & 10,6900 & 0,0001 \\
$\quad$ Acidez (G) & 4 & 330,1351 & 82,5338 & 1,6000 & 0,1834 \\
Acidez (SS) & 4 & 1169,2233 & 292,3058 & 5,6600 & 0,0005 \\
Acidez (ST) & 4 & 147,1985 & 36,7996 & 0,7100 & 0,5856 \\
Acidez (Doses x Aditivos) & 12 & 1646,5568 & 137,2131 & 2,6585 & 0,0050 \\
\hline Resíduo & 75 & 3870,9259 & 51,6123 & & \\
\hline Total & 99 & 22131,2491 & & & \\
\hline
\end{tabular}

TABELA 7 - Análise da variância apresentando efeito linear, quadrático e cúbico para doses de superfosfato triplo, e também, o efeito da acidez residual na presença das doses do superfosfato simples.

\begin{tabular}{lcrrrr}
\hline Fonte de Variação & G.L. & \multicolumn{1}{c}{ S.Q. } & \multicolumn{1}{c}{ Q.M. } & \multicolumn{1}{c}{ F } & p-valor \\
\hline Doses linear (ST) & 1 & 1,1526 & 1,1526 & 0,02 & 0,8816 \\
Doses quadrático (ST) & 1 & 1230,2137 & 1230,214 & 23,84 & 0,0001 \\
\hline Doses cúbico (ST) & 1 & 104,4735 & 104,473 & 2,02 & 0,1590 \\
\multicolumn{1}{c}{ Doses (ST) } & 3 & 1335,8398 & 445,2799 & 8,63 & 0,0001 \\
Acidez (50 SS) & 1 & 6,8821 & 6,8821 & 0,13 & 0,7160 \\
Acidez (100 SS) & 1 & 947,8658 & 947,8658 & 18,37 & 0,0001 \\
Acidez (150 SS) & 1 & 178,6050 & 178,6050 & 3,46 & 0,0668 \\
Acidez (200 SS) & 1 & 35,8704 & 35,8704 & 0,69 & 0,4071 \\
\multicolumn{1}{c}{ Acidez (SS) } & 4 & 1169,2233 & 292,3058 & 5,66 & 0,0005 \\
Resíduo & 75 & 3870,9259 & 51,6123 & & \\
\hline Total & 99 & 22131,2491 & & & \\
\hline
\end{tabular}




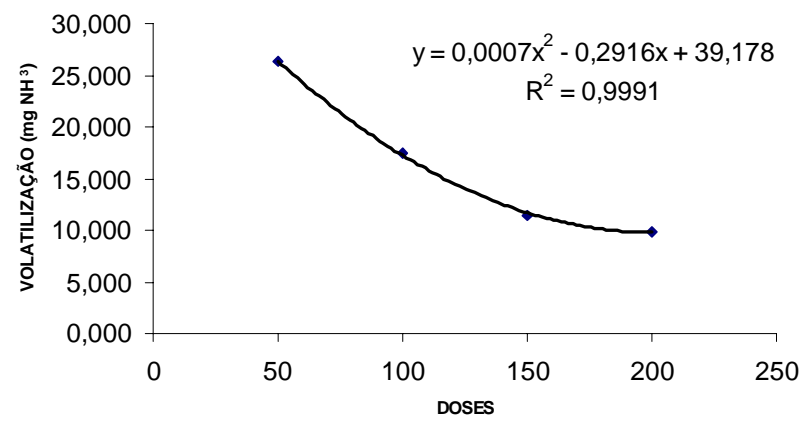

Figura 1 - Análise de regressão da volatilização $\left(\mathrm{mg} \mathrm{NH}_{3}\right)$ da amônia em função de doses de superfosfato triplo.

revelando assim que para menor acidez residual ocorre maior volatilização da amônia, como pode ser observado através dos valores médios estimados para a dose 100 $\mathrm{kg}$ ton $^{-1}$ de superfosfato simples para a acidez residual de $7,02 \%$ e $2,36 \%$.

\section{CONCLUSÃO}

A aplicação de contrastes ortogonais revelou uma alternativa de análise eficiente para a estimação dos efeitos de interesse, quando os dados seguem uma estrutura não-convencional. Esta técnica permitiu um maior grau de detalhamento da análise e tornou possível testar os vários efeitos envolvidos e de interesse.

\section{AGRADECIMENTOS}

Ao Prof. Dr. Luis Ignácio Prochnow do Departamento de Solos e Nutrição de Plantas da USP/ ESALQ, pela cessão dos resultados do experimento de volatização.

\section{REFERÊNCIAS BIBLIOGRÁFICAS}

ALCARDE, J.C.; RODELLA, A.A. O equivalente em carbonato de cálcio dos corretivos da acidez do solo. Scientia Agricola, v.53, p.204-210, 1996.

ALEXANDER, M. Introduction to soil microbiology. 2.ed. New York: John Wiley, 1977. 467p.

GALBALLY, I.E.; ROY, C.R. The fate of nitrogen compounds in the atmosphere. In: FRENEY, J.R.; SIMPSON, J.R. (Ed.) Gaseous loss of nitrogen from plant-soil systems. Hague: Martinus Nijhoff, 1983. p. 265-284.

GOMES, F.P. Curso de estatística experimental. 12.ed. São Paulo: Livraria Nobel, 1987. 403p.

HEALY, M.J.R. The analysis of a factorial experiment with additional treatments. The Journal of Agriculture Science, v.47, p.205-206, 1956.

MOLLER, D.; SHIEFERDECKER, H. A relationship between agricultural $\mathrm{NH} 3$ emissions and the atmospheric $\mathrm{SO} 2$ content over industrial areas. Atmospheric Environment, v.19, p.695-700, 1985.

PROCHNOW, L.I.; CUNHA, C.F.; KIEHL, J.C.; ALCARDE, J.C. Controle da volatilização de amônia durante a compostagem, através da adição de superfostato simples, superfosfato triplo e gesso agrícola com diferentes níveis de acidez residual. In: REUNIÃO BRASILEIRA DE FERTILIDADE DO SOLO E NUTRIÇÃO DE PLANTAS, 23., Caxambú, 1998. Resumos. Lavras: UFLA, SBCS, SBM, 1998.

SAS INSTITUTE. SAS/STAT user's guide. 4.ed. Cary: Statistical Analysis System Institute, 1990. 1675p.

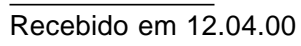

